\title{
Low energy excited state vibrations revealed in conjugated copolymer PCDTBT
}

Cite as: J. Chem. Phys. 152, 044201 (2020); https://doi.org/10.1063/1.5132299

Submitted: 16 October 2019 . Accepted: 30 December 2019 . Published Online: 22 January 2020

Shawn Irgen-Gioro (D), Palas Roy (D), Suyog Padgaonkar, and Elad Harel (D)

\section{COLLECTIONS}

Paper published as part of the special topic on Ultrafast molecular sciences by femtosecond photons and electrons

Note: This paper is part of the JCP Special Topic on Ultrafast Molecular Sciences by Femtosecond Photons and Electrons.
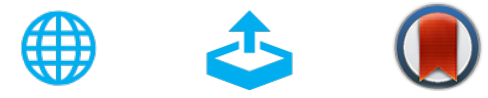

\section{ARTICLES YOU MAY BE INTERESTED IN}

Benchmark ab initio calculations on intermolecular structures and the exciton character of poly(p-phenylenevinylene) dimers

The Journal of Chemical Physics 152, 044306 (2020); https://doi.org/10.1063/1.5139411

Lessons from intramolecular singlet fission with covalently bound chromophores

The Journal of Chemical Physics 152, 040904 (2020); https://doi.org/10.1063/1.5135307

The density matrix renormalization group in chemistry and molecular physics: Recent developments and new challenges

The Journal of Chemical Physics 152, 040903 (2020); https://doi.org/10.1063/1.5129672

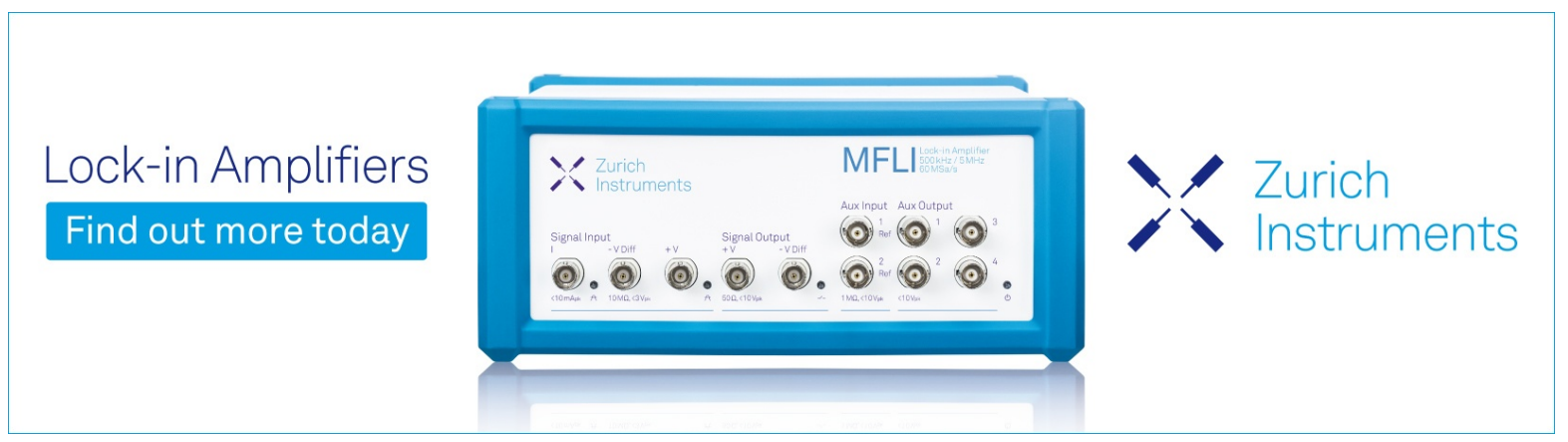




\title{
Low energy excited state vibrations revealed in conjugated copolymer PCDTBT
}

\author{
Cite as: J. Chem. Phys. 152, 044201 (2020); doi: 10.1063/1.5132299 \\ Submitted: 16 October 2019 - Accepted: 30 December 2019 • \\ Published Online: 22 January 2020
}

\author{
Shawn Irgen-Gioro,' (D) Palas Roy, ${ }^{1,2}$ (D) Suyog Padgaonkar,' and Elad Harel ${ }^{1,3, a)}$ (D)
}

\begin{abstract}
AFFILIATIONS
${ }^{1}$ Department of Chemistry, Northwestern University, 2145 Sheridan Rd. Evanston, Illinois 60208, USA

${ }^{2}$ School of Chemistry, University of East Anglia, Norwich Research Park, Norwich, NR4 7TJ, United Kingdom

${ }^{3}$ Department of Chemistry, Michigan State University, East Lansing, Michigan 48824, USA
\end{abstract}

Note: This paper is part of the JCP Special Topic on Ultrafast Molecular Sciences by Femtosecond Photons and Electrons.

a) Author to whom correspondence should be addressed: elharel@msu.edu

\begin{abstract}
Low energy vibrations in the excited state have been hypothesized to play an important role in quickly and efficiently generating free charges in bulk heterojunctions of some conjugated polymer systems. While time-resolved vibrational spectroscopies seemingly are well poised to address the relationship between kinetics and vibrational motions after initial photoexcitation, uncertainty in the measurement arises due to overlapping signals and difficulties in assigning observed oscillatory signals to the molecular response. Here, we demonstrate a high sensitivity strategy to distinguish between signal oscillations originating from lab noise and those molecular in origin in order to isolate the low energy excited-state vibrations in the model conjugated copolymer PCDTBT. Furthermore, to distinguish modes that may be implicated in different kinetic pathways, coherent signal oscillations extracted from 2-dimensional electronic spectroscopy (2DES) are compared for the polymer in two solvents with different polarities resulting in different kinetics. We observe that the change in solvent affects dynamics on the $>2$ ps scale but not on the time scale required for free charge generation in heterojunctions ( $200 \mathrm{fs}$ time scale). By the same token, the excited state vibrational modes that appear and disappear based on solvent polarity may also be associated with the slower kinetic process. The observation of low energy vibrational motions coupled to the excited state manifold that persists through the solvent change and thus can be associated with the fast kinetic process supports the hypothesis that direct polaron formation, rather than exciton formation and diffusion followed by interfacial charge separation, is a more likely route toward free charges in organic heterostructures.
\end{abstract}

Published under license by AIP Publishing. https://doi.org/10.1063/1.5132299

\section{INTRODUCTION}

Owing to their high internal efficiency, strong visible absorption, and economic viability, conjugated donor-acceptor copolymers are being considered as possible low-cost alternatives to silicon photovoltaic devices acting as light absorbers and electron donors. When blended with electron acceptors such as fullerene into an organic bulk heterojunction, mobile free charges appear faster than is predicted by traditional models in which excitons diffuse to the interface and form interfacial charge separated states. ${ }^{2-4}$ While this speed helps explain the efficiency and, in turn, the performance of these materials in a photovoltaic context, the mechanism utilized in route to generating free charges is under debate. ${ }^{5,6}$ The disparity in the time scales initially prompted researchers to suggest that the transport is at least partially coherent, which could increase exciton diffusion lengths and the transport rate. In highly structured and perfectly ordered pi-conjugated chains, strong intrachain electronic coupling leads to extended delocalization where transport occurs stochastically by means of population relaxation. ${ }^{7}$ However, many polymers demonstrating fast long-range transport are amorphous and have a significant degree of disorder. While disorder may limit the extent of delocalization, this is only the case for isolated electronhole pairs or excitons. Polarons, quasiparticles that result from coupling of the charges with the geometric motion of the polymer chain, may help facilitate free charge generation by utilizing this disorder to their advantage. ${ }^{6,8-11}$ These amorphous polymer systems exist in an intermediate coupling regime in which the geometric relaxation energies are on the same order of magnitude as the electronic coupling. ${ }^{5}$ This leads to an interplay of delocalization and localization where collective jumps in excitation that are delocalized over many chromophores reduce the number of diffusive steps required, sometimes referred to as "supertransfer." 12 
Coupling of electronic motion to vibrational modes is intrinsic to conjugated polymers since the lowest energy transition involves the same $\pi$ orbitals that delocalize electron density along the polymer chain. Any modification to these bonds (C-C stretching or ring breathing modes) will modify the $\pi-\pi^{*}$ electronic transitions. These vibrational energies are typically in the thousands of wavenumbers regime and are relevant to intrasegment dynamics. ${ }^{13}$ Electronphonon coupling in this higher energy regime is typically more experimentally accessible using methods such as Femtosecond Stimulated Raman Spectroscopy (FSRS) and Infrared-Active Vibrational (IRAV) spectroscopy, and previous studies have successfully correlated structural changes to polaron formation. ${ }^{14-17}$ In the case of amorphous polymers, charge carriers need to jump between chain segments. This intersegment interaction has weaker wavefunction overlap, leading to coupling strengths in the $50-500 \mathrm{~cm}^{-1}$ regime, where a different set of vibrational modes are relevant. Recent work has proposed that polarons and excitons are vibronically coupled, resulting in the direct generation of polarons rather than mobile charges that follow from the disassociation of excitons. ${ }^{18}$ Other studies have proposed that vibrations that are strongly coupled to excitons create a high density of states that entropically favor electron and hole hopping between subunits rather than electron-hole attraction. ${ }^{19-23}$ This high density of states is also thought to explain the weak correlation between energy-level alignment of exciton states and power conversion efficiency of organic photovoltaic devices. ${ }^{5,24}$ Natural multichromophoric photosynthetic complexes have demonstrated similar strategies to increase transport lengths in the presence of structural disorder that may be applied to electronic-vibrational coupling in conjugated polymers. In particular, studies show that a high density of vibronic states along with vibrations equal to the energy gap between electronic states result in a resonant condition conducive to fast transfer. $^{25,26}$

While it is clear that the characterization of transient low energy vibrations on the electronic excited state is important, access to this energy regime is difficult experimentally. Raman based techniques have an inherent trade-off between time resolution and the ability to observe low frequency modes, and direct detection of IR-active modes requires generation of far-IR light, which is extremely challenging below $\sim 500 \mathrm{~cm}^{-1}$. Instead of using narrowband pulses to generate population in the excited state, techniques such as impulsive vibrational spectroscopy or 2-dimensional spectroscopy (2DES) use compressed broadband light to simultaneously track populations and coherences, which report on both the kinetics and vibrations in the time domain. However, since vibrations are detected as fluctuations of the signal in time, periodic lab noise can be mapped onto the signal and may cast doubt onto whether the detected oscillations are physically meaningful. Furthermore, even when a signal is confirmed to arise from a molecular vibration, assignment of how that vibration couples to any particular electronic state is ambiguous. Here, we develop a method which we call Variable Bandwidth Noise Minimization (VBNM) to separate out signals from the system of interest from parasitic signals that arise from laboratory noise lab frame. To further isolate the

a.

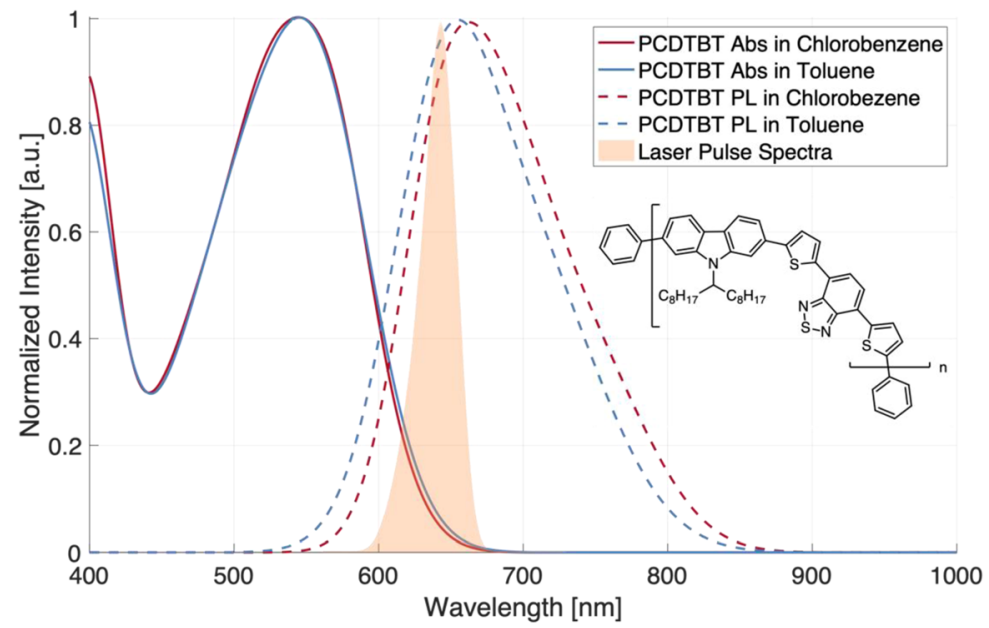

b. CEES of PCDTBT in $\mathrm{CB}$

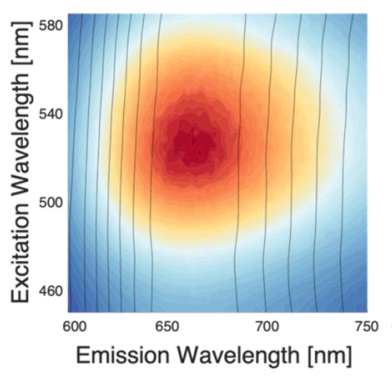

C. CEES of PCDTBT in Tol

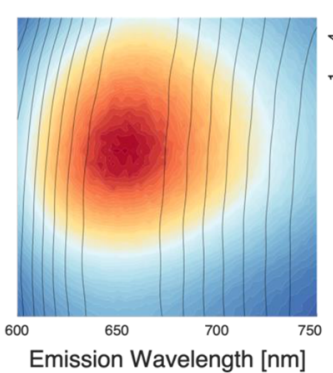

d. 2DES of PCDTBT in $\mathrm{CB}$

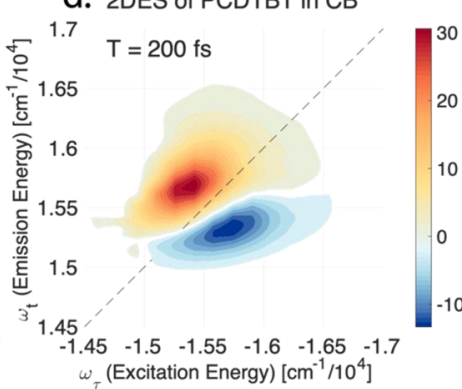

FIG. 1. (a) Absorption and photoluminescence (excited at absorption maximum) of PCDTBT in both chlorobenzene and toluene. Combined Excitation Emission Spectra (CEES) of PCDTBT in chlorobenzene (b) and toluene (c). Contour lines drawn from emission spectra normalized at each emission peak. This helps show that in chlorobenzene (CB) PCDTBT's emission spectra's peak position is independent of excitation energy, while in toluene (TL), excitation of the inhomogeneous broadened absorption spectra yields emission from distinct subpopulations. (d) Slice of 2DES spectra at a fixed $T=200 \mathrm{fs}$ along dimensions popularly interpreted as excitation and emission dimensions. Positive features represent either ground state bleach or stimulated emission features while negative features represent excited state absorption (ESA). Our spectra are in good agreement with previous work by the Scholes group who detailed the dynamics in Ref. 9 and assigned the ESA to the polaron state formed in $\sim 200 \mathrm{fs}$. Our study instead focuses on coherences observed as oscillations of the signal as a function of $T$. 
mechanism of carrier generation, kinetic pathways are distinguished through the variation of solvent polarity, which in turn allows for assignment of vibrations to a particular dynamic process. ${ }^{27}$ For solvents without a strong hydrogen bonding network, the change of dielectric constants can be understood within the framework of the Stark effect, meaning that changes in transition energies are related to the difference of a particular state's permanent dipole moment and polarizability in the two solvents. ${ }^{28,29}$ Leveraging the fact that copolymer's charge transfer states have separated charges leading to large electric dipole moments $(\mu=q d)$ amenable to change, vibrations associated and not associated with that kinetic pathway can be separated out by comparing the coherences observed in different solvents.

Our study focuses on one of the well-celebrated conjugated "push-pull" polymers, which alternates between electron rich and deficient units, PCDTBT within the poly(2,7-carbazole) family synthesized by Leclerc et al. as shown in Fig. 1(a). ${ }^{30}$ Exhibiting high stability in air, photovoltaic cells fabricated with a PCDTBT blend active layer may achieve photoconversion efficiencies beyond $6 \%$, and internal quantum efficiencies near $100 \%$ have been reported. ${ }^{3,31,32}$ Both the absorption and fluorescence of PCDTBT in chlorobenzene and toluene are shown in Fig. 1(a). The lowest energy absorption peak has been assigned to a localized charge transfer transition, in which the electron density is concentrated at several electron-accepting units, which are themselves distributed over the chain. ${ }^{33}$ The transition is inhomogeneous broadened, arising from the different torsion, coiling, and folding configurations of the backbone. Previous work has documented excited state dynamics following excitation of this transition tracked through ENDOR, 2DES, fluorescence upconversion, and Transient Absorption (TA) spectroscopies, but distinguishing between different kinetic processes after photoexcitation has been challenging. ${ }^{2,6,34,35}$ Provencher et al. doped a PCDTBT film and found that the polarons exhibit a broad absorption stretching from $\sim 1.2$ to $2.0 \mathrm{eV}(620-1030 \mathrm{~nm})$ peaking at $1.7 \mathrm{eV}(\sim 730 \mathrm{~nm})$ assigned to be from interband transitions. ${ }^{10}$ However, since other excited species also have absorption in the same region, other handles such as kinetic rates or vibrational peak amplitudes have been used to track polaron population. ${ }^{2}$ While the exact dynamics after photoexcitation is unknown, the fact that free charges are generated within $\sim 200$ fs in the polymer heterostructures has led some to suggest that local structural lattice distortions of the copolymer directly self-localize charges as polarons without needing to diffuse to the interface's charge transfer state. ${ }^{2,6,33} 2 \mathrm{DES}$ performed on this system by the Scholes group tracked peak energies of ground state bleach (GSB) and excited state absorption (ESA) features to observe internal relaxation on a $\sim 200 \mathrm{fs}$ time scale that they assigned geometric changes but did not directly observe any excited state vibrations in the form of coherences.

\section{RESULTS}

PCDTBT is studied in two aromatic solvents, toluene and chlorobenzene, to examine the effect of solvent polarity on the generation of polarons and charge transfer states. A combined excitation and emission (CEES) plot is displayed in Fig. 1(b), which shows the fluorescence spectra of PCDTBT in chlorobenzene as a function of excitation wavelength. The emission spectra are consistent, independent of the excitation wavelength. However, when the same measurement is performed for PCDTBT in toluene [Fig. 1(c)], the fluorescence peak shifts as a function of the excitation energy. Since the absorption is primarily inhomogeneously broadened, changing excitation wavelengths photoselects for differently twisted subpopulations resulting in a shifting fluorescence peak. In chlorobenzene, this effect is not seen due to the fact that all the excited species relax to a common untwisted planar configuration, a key result from the Scholes group report. ${ }^{9}$ Another key result from the previous 2DES study was that an excited state absorption (ESA) was observed to
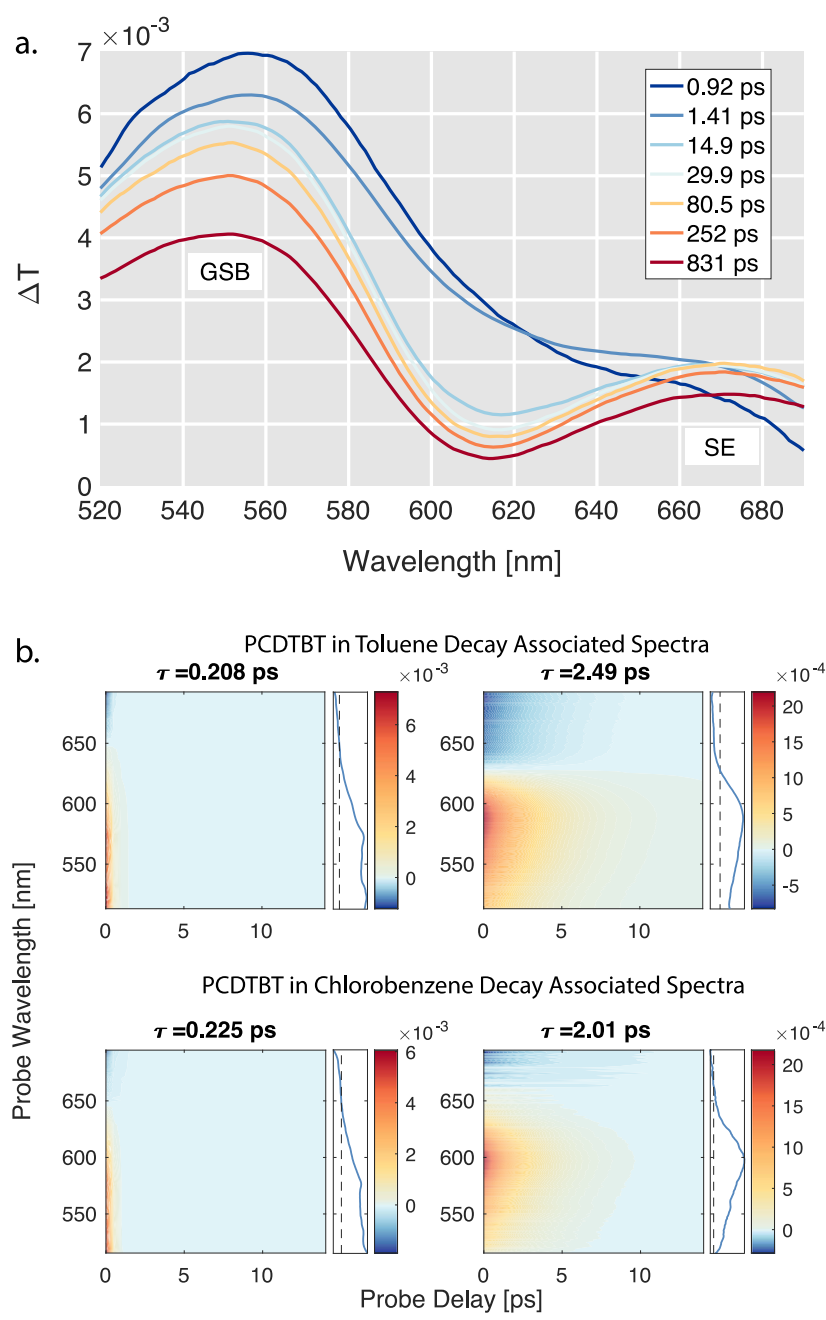

FIG. 2. Transient absorption of PCDTBT shown as a function of probe wavelength and probe delay. (a) Immediately after excitation, two peaks, assigned to be ground state bleach and stimulated emission, are observed. (b) Global analysis is used to recover the decay associated spectra (DAS). Only the two fastest components are shown here, but they can be used to illustrate the fact that the fastest $200 \mathrm{fs}$ component is agnostic to the choice of solvent. However, the change in solvent polarity changes the energy in which the signal rises on a $\sim 2$ ps time scale. This is more clearly seen in the inserts to the right of the DAS, where the zero crossing point for the 200 fs component does not change between solvents, but the zero crossing in the $\sim 2$ ps component shifts to the red in chlorobenzene. This establishes that processes happening faster than 2 ps are insensitive to solvent change. 
appear within $200 \mathrm{fs}$. A slice of the 2DES measured in our experiment at a fixed population time of $200 \mathrm{fs}$ is shown in Fig. 1(d) and shows a negative ESA feature to the Stokes side of the diagonal, the same energy as the polaron absorption and in agreement with the previous 2DES study. ${ }^{16}$ The same time slice in both solvent does not show differences in peak energies, suggesting that the ESA feature is insensitive to solvent change at early times.

Transient absorption (TA) spectroscopy is performed to examine the changes to the kinetic pathways of the polymer in the two solvent environments. Using a narrow-band pump wavelength of $500 \mathrm{~nm}$, a broadband probe is used to examine the energy region where the absorption, fluorescence, and polaron state transitions exist. The TA spectra for the polymer in chlorobenzene are shown in Fig. 2(a) and show that immediately after excitation, two peaks around $550 \mathrm{~nm}$ and $670 \mathrm{~nm}$ appears. These can be assigned to be the ground state bleach (GSB) and stimulated emission (SE), respectively, from the steady state absorption and fluorescence spectra. The stimulated emission peak grows in and shifts toward lower energies as probe delay increases. In order to better characterize the transfer processes and the state energies involved, global analysis was performed. Figure 2(b) displays the result of this fitting, which deconstructs the original dataset into a set of spectra each containing only either a positive or a negative amplitude exponential with the same time constant that can be summed to reconstruct the original data. Each of these Decay Associated Spectra (DAS) readily shows the probe energy at which the signal either rises or decays at the same rate, with a positive amplitude corresponding to a decay of signal while a negative amplitude exponential represents a growth of signal. While only two components are displayed for the scans in both solvents, the full set of dynamic components can be found in the supplementary material (SI) along with the reconstructed dataset to be compared with the original data. Comparing the fastest two dynamic components of the DAS of PCDTBT in the two solvents show that the component with a time constant of $\sim 200 \mathrm{fs}$ is similar for both scans. However, a comparison of the $\sim 2$ ps components shows that the growth of signal is centered at a lower energy for chlorobenzene. This may be more readily seen in the inset to the right of the 2D DAS plots, which show that the zero-crossing point for the DAS is the same for the $200 \mathrm{fs}$ component but shifts to the red for the $\sim 2$ ps component. Since chlorobenzene is the more polar solvent, the intramolecular charge transfer state should be more stabilized in this environment. We thus assign the slower 2 ps component to the exciton mediated intramolecular charge transfer process, but no processes faster, namely, the polaron formation, were affected by the solvent change.

As opposed to the narrow-band pump in TA, 2DES's use of a broadband pump is capable of creating vibrational coherences and thus detecting excited state vibrations as oscillations of the signal as a function of population time. In this methodology, broadband compressed laser pulses initiate vibrations on the ground and excited states that are observed as oscillations as a function of delay between laser pulses and Fourier transformed into the frequency domain. However, time-domain measurements are susceptible to different sources of noise than frequency-domain techniques, which in turn casts doubt on the reliability of low energy peaks. Previous work in our group has demonstrated how to reduce phase and amplitude pink (1/f) noise through a combination of techniques to increase acquisition speed and stability, but narrow-band noise (ex. $60 \mathrm{~Hz}$ electrical noise, periodic flow of sample, mechanical vibrations, etc.) persists. ${ }^{36,37}$ While pink noise may hinder observation of coherences,
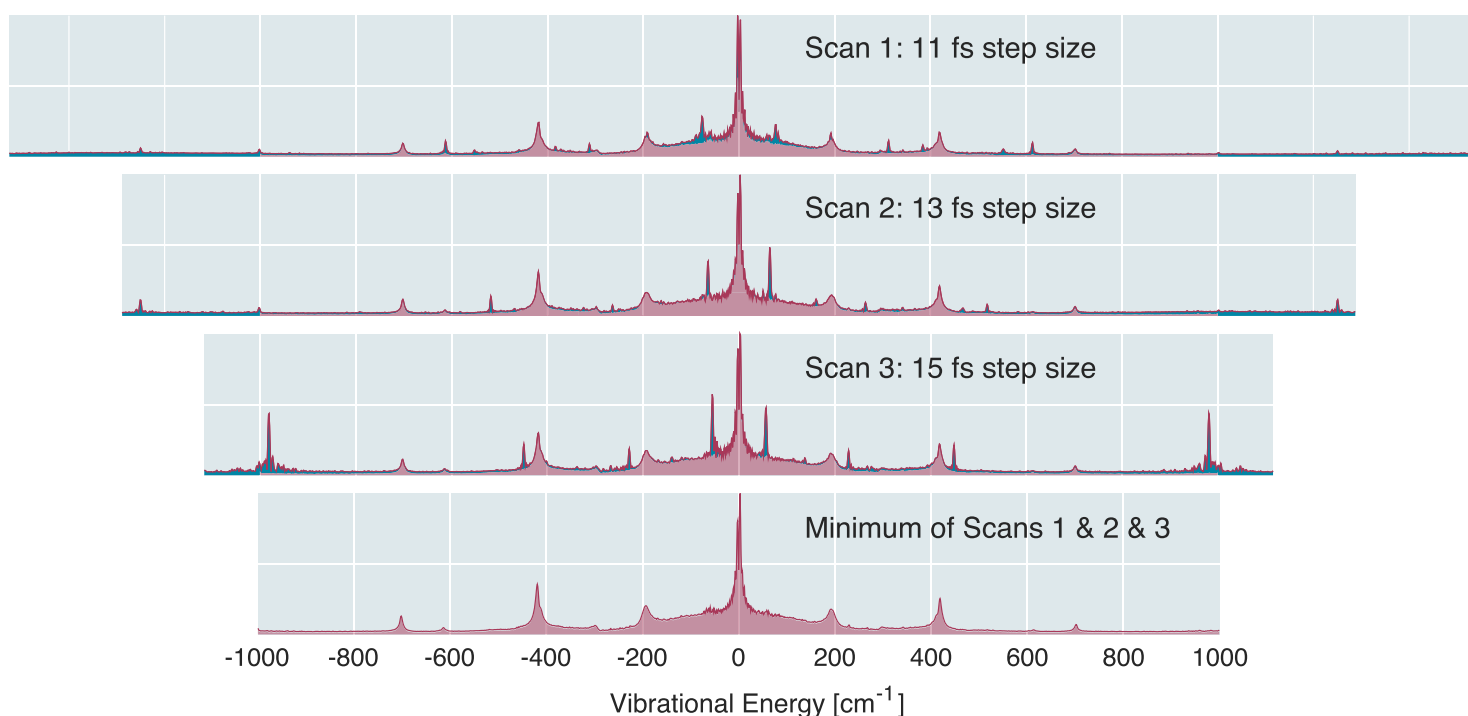

FIG. 3. Experimental demonstration of the Variable Bandwidth Noise Minimization (VBNM) scheme for time resolved vibrational measurements. Due to the fact that molecular response is insensitive to the bandwidth of measurement (given the signal is not being aliased) while narrow-band noise scales along with bandwidth, the two types of oscillation can be separated out by taking scans with different step sizes. Comparing the scan at a set step size vs the minimization of three scans with different step sizes, peaks that may have been misinterpreted as molecular response are removed. The minimized scan is overlaid on the differing step size scans, highlighting which peaks persist (in red) and which get removed through this minimization procedure (blue). 
narrow-band noise is potentially more troublesome as it casts doubt on whether observed peaks are real. This ambiguity has hampered the broad application of time domain vibrational spectroscopy in the case of weak signals at low-frequency. Here, we introduce a procedure to vary the bandwidth of measurement to isolate the molecular response.

The difference between oscillations arising from molecular vs instrument response hinges on the fact that the molecular response depends on the time delay between laser pulses and instrument

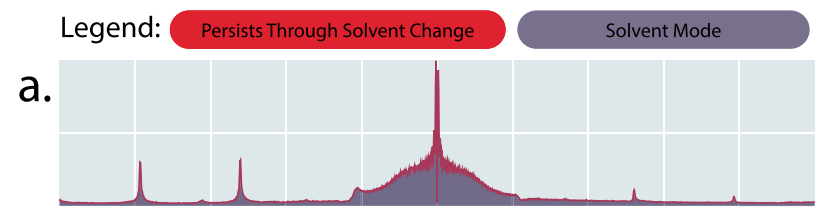

b.

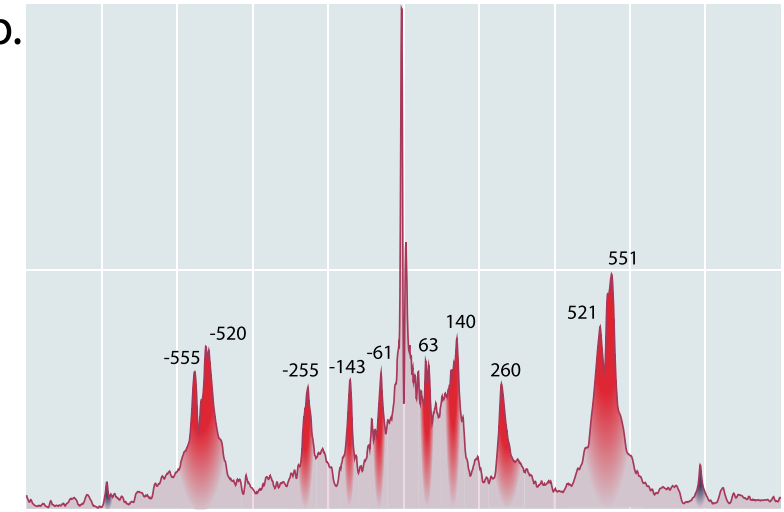

C.
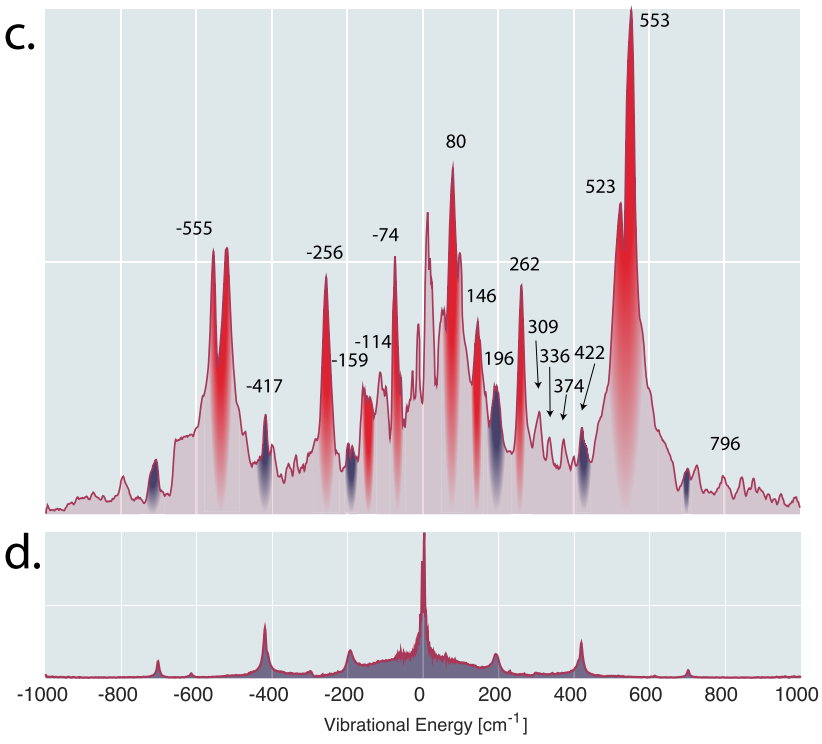

FIG. 4. Coherences measured in (a) toluene only, (b) PCDTBT in toluene, (c) PCDTBT in chlorobenzene, and (d) chlorobenzene only. Modes associated with the solvent are colored in blue, while peaks that are persist through the solvent change are marked in red. Since the solvent change affected only slower kinetic pathways, the modes unaffected by the solvent change are hypothesized to be involved in the generation of polarons. response depends on events happening in lab time. Therefore, if the time step between laser pulses is varied, the molecular vibrational frequencies should not change (given they are not being aliased). However, the oscillations introduced by lab noise show up at different frequencies. This VBNM procedure is demonstrated experimentally on a nonresonant scan of chlorobenzene in Fig. 3. As the step size of the scan is varied, certain peaks stay constant, while others change along with the bandwidth based on the Nyquist criteria. To isolate the peaks that are constant, taking the minimum value of a frequency between scans ensures that any peak that is not constant between all the scans is removed. Comparing the minimization of the scans of 11,13 , and $15 \mathrm{fs}$ steps to the individual scans, it can be seen that many peaks have been removed that may have previously been incorrectly assigned to ground state modes of chlorobenzene. Of course, this procedure relies on the fact the noise peaks can be shifted significantly (more than the noise peak width). Thus, although the noise peaks can be shifted through other means (such as changing the acquisition speed of the camera), we find that changing the step size allows for the greatest noise peak shifts without negatively affecting the signal-to-noise ratio (SNR). This technique works well by simply minimizing two scans, but additional scans allow for averaging between scans. Although not implemented in this study, previous work has shown additional SNR benefits of averaging combinations of different scans before taking the lowest value.

With confidence in the reliability of the low energy coherences, the VBNM procedure is applied in 2DES of toluene only, PCDTBT in toluene, PCDTBT in chlorobenzene, and chlorobenzene only, which are displayed in Figs. 4(a)-4(d). The assignment of these modes to the excited state is confirmed comparing the coherences to nonresonant Raman as well as identifying the Liouville pathways examined in the experiment, discussions of which may be found in the supplementary material. Taking the scan in two different solvents allows for modes of the polymer to be separated from solvent modes as well as distinguish vibrations associated with a specific kinetic process. Any peak that appears and disappears is associated with the slower kinetic processes that were sensitive to the solvent change. The color coding helps guide the interpretation as peaks colored in blue are assigned to be from the solvent, while the peaks in red are the ones that persist between the solvent change.

\section{DISCUSSION}

The VBNM and change of solvent both are general strategies to filter out congested spectra applied in our case to associate excited state vibrations to particular kinetic processes. The TA and fluorescence measurements demonstrate that the more polar chlorobenzene solvent stabilizes the charge transfer state of PCDTBT and affects kinetics on a time scale longer than $\sim 2$ ps. On the other hand, the time scale that free charges are generated in heterojunctions is not affected by the solvent change. This observation along with the fact that the fast time scale is associated with excited state vibrational modes leads us to assign this early kinetic process to direct polaron formation, a mechanism described in P3HT 2DES studies. ${ }^{18}$ This paints the picture that the generation of polarons and the population of intramolecular charge transfer states are two competitive kinetic 
pathways. Observation of both electronic and vibrational spectral signatures of polarons directly after photoexcitation makes it seem likely that polarons are the likely source of ultrafast free carriers in heterojunctions. ${ }^{6,18,39}$

Our study identifies a set of vibrations that are coupled to the lowest excited state transition that may be involved in polaron formation. In particular, the modes with energy $<100 \mathrm{~cm}^{-1}, \sim 140 \mathrm{~cm}^{-1}$, $\sim 260, \mathrm{~cm}^{-1}-520 \mathrm{~cm}^{-1}$, and $\sim 550 \mathrm{~cm}^{-1}$ all can be assigned to motions of the polymer and persisted through the solvent change. The $<100 \mathrm{~cm}^{-1}$ mode that shows up in toluene at $\sim 60 \mathrm{~cm}^{-1}$ and in chlorobenzene at $\sim 80 \mathrm{~cm}^{-1}$ are of particular interest as half a period of their vibration is in the $200 \mathrm{fs}-300 \mathrm{fs}$ range. This half period is important because this is the time scale in which the vibration reaches its maximum displacement representing the time scale at which the phonon or molecular structure lags behind. Once the phonon catches up and localizes the charge, electron-hole attraction is greatly reduced thus suppressing recombination. Thus, the time scale of polaron formation and the period of these $<100 \mathrm{~cm}^{-1}$ modes suggest this mode as a good candidate for the reaction coordinate for polaron formation. However, without more interpretation of what physical motions each of the identified modes correspond to, it is difficult to unequivocally isolate which modes are important. What is clearly shown is that the excited state of this highly efficient conjugated polymer studied is coupled to many low energy vibrational motions. Understanding the differing role each of these modes play will be an area of future research.

Previous reports of coherences from 2DES have utilized the pattern in which coherences show up in their spectrum, known as beat maps, to assign the origin of the coherences to either the exciton, charge transfer state, or the polaron. ${ }^{18,40}$ Beat maps for coherences found in our study can be found in the supplementary material. However, broad overlapping features arising from the high density of states of the polymer system along with our room temperature measurement complicate the assignment of coherences originating from particular excited states.

\section{CONCLUSION}

Utilizing new experimental techniques to suppress laser and laboratory noise, our study reports on low energy vibrations coupled to the excited state of the conjugated polymer PCDTBT. A comparison of PCDTBT in two solvents reveals that kinetic processes occurring on a $>2$ ps time scale are affected by the solvent polarity, while the $\sim 200$ fs time scale of polaron formation is not. This information is applied to the study of the excited state vibrations of the polymer, where certain coherences disappear and appear in the different solvent environments, while modes associated with the polaron should remain. Identification of modes likely associated with polaron formation helps to untangle the complicated excited state landscape. This methodology to assign vibrational function by changing chemical parameters will be explored further in future research.

\section{METHODS}

\section{Spectroscopic data collection}

CEES data were taken on the photon-counting fluorescence spectrometer (PC1 by $\mathrm{ISS}^{\mathrm{TM}}$ ). Absorption spectra are taken on a
Shimadzu UV-1800 spectrometer. PL data displayed in Fig. 1(a) are simply a single row of the CEES chosen with a $545 \mathrm{~nm}$ excitation wavelength. Raman data were collected using a Horiba LabRAM HR Evolution with $785 \mathrm{~nm}$ excitation and $600 \mathrm{gr} / \mathrm{mm}$. Samples were in taken in a $2 \mathrm{~mm}$ quartz cuvette with an integration time of $400 \mathrm{~s}$ with 2 accumulations to account for stray cosmic rays.

Transient absorption was collected using a commercial Helios system described in detail in the supplementary material of Ref. 41. The pump energy used was $1 \mathrm{~mW}$ of $1 \mathrm{kHz}$ light at $500 \mathrm{~nm}$ generated by an optical parametric amplifier (TOPAS-C from Light Conversion). Probe delays were taken out to $3 \mathrm{~ns}$.

The spectroscopic setup used to collect the 2DES has been described in detail previously in Ref. 36. Briefly, broadband pulses are generated from a 3rd harmonic non-collinear optical parametric amplifier (3H-NOPA) (ORPHEUS-N) seeded by a $200 \mathrm{kHz}$ $1028 \mathrm{~nm}$ laser (Pharos) compressed with a prism compressor and measured using transient grating frequency-resolved optical gating (TG-FROG) at the sample position with solvent (pulse characterization found in supplementary material). The spectrometer is built in a passively phase stable configuration, and the signal is detected using Gradient Assisted Photon Echo Spectroscopy (GRAPES). Since GRAPES encodes $\tau$ delays spatially and $\omega_{t}$ is resolved using a spectrometer, the only delay to be scanned is T. This delay is scanned continuously without stopping the stage allowing for a full $3 \mathrm{D}$ dataset to be collected in $<2 \mathrm{~s}$ lab time. This rapid acquisition beats out much of the $1 / \mathrm{f}$ noise. In order for the scans with different step sizes to be correlated, no parameters are changed between different scans other than the step size. The phase of the complex signal is retrieved through spectral interferometry. Interference with a local oscillator allows for retrieval of a relative phase. The phase of the nonresonant signal is known to be purely dispersive, so the relative phase of the nonresonant signal to the local oscillator is used to roughly phase the PCDTBT signal. The phase is fine tuned by making sure that the $2 \mathrm{DES}$ signal summed along $\omega_{\tau}$ matches the pump probe signal according to the projection-slice theorem. Verification of the pump probe signal relative to the sum of the 2DES signal can be found in the supplementary material.

\section{Variable bandwidth procedure}

The signal obtained with different step sizes yields different bandwidths along $\omega_{T}$ but the same range of $\omega_{\tau}$ and $\omega_{t} . \omega_{T}$ is first obtained for each $\omega_{\tau}$ and $\omega_{t}$ by removing the population dynamics through a global analysis procedure described in Ref. 36 and then Fourier transforming the residual. The largest step size determines the bandwidth relevant for the minimization procedure. All three scans are interpolated onto a new $\omega_{T}$ axis (or simply the $\omega_{T}$ axis of the largest step size scan). For each point in $\omega_{\tau}$ and $\omega_{t}$, the different scans now with the same number of points along $\omega_{T}$ are minimized with respect to each other. To get the figures shown in Figs. 3 and $4(\mathrm{a})-4(\mathrm{~d})$, the minimized $3 \mathrm{D}$ data cube is summed along $\omega_{\tau}$ and $\omega_{t}$. This procedure relies on a high degree of correlation between scans of different step sizes. Thus, it is critical that no parameters of the scan are changed other than the step size.

\section{Sample preparation}

PCDTBT was bought from Sigma-Aldrich (Mw $20000-$ $100000)$ and used without further purification. Previous 2DES 
measurements of PCDTBT were limited by scatter due to formation of large aggregates at higher concentration. We found that starting with low concentrations and slowly adding in solute to reach relatively high concentrations of PCDTBT removes scattering. For the 2DES measurement of PCDTBT in chlorobenzene had an optical density of 0.19 in a $200 \mu \mathrm{m}$ quartz flow cell and 0.18 in toluene.

\section{SUPPLEMENTARY MATERIALs}

See the supplementary material for additional figures, pulse characterization, and discussion of excited state vs ground state coherence

\section{ACKNOWLEDGMENTS}

The authors thank the Keck facility, especially Dr. Ronald Soriano, at Northwestern University for his help with the CEES measurement. This work made use of the Keck-II facility of Northwestern University NUANCE Center, which has received support from the Soft and Hybrid Nanotechnology Experimental (SHyNE) Resource (No. NSF ECCS-1542205); the MRSEC program (No. NSF DMR-1720139) at the Materials Research Center; the International Institute for Nanotechnology (IIN); the Keck Foundation; and the State of Illinois, through the IIN. This work was supported by the Air Force Office of Scientific Research (Grant No. FA9550-141-0005) and the Packard Foundation (Grant No. 2013-39272) in part.

\section{REFERENCES}

${ }^{1}$ N. S. Lewis, "Toward cost-effective solar energy use," Science 315, 798 (2007).

${ }^{2}$ F. Etzold et al., "Ultrafast exciton dissociation followed by nongeminate charge recombination in PCDTBT:PCBM photovoltaic blends," J. Am. Chem. Soc. 133, 9469-9479 (2011).

${ }^{3}$ S. H. Park et al., "Bulk heterojunction solar cells with internal quantum efficiency approaching 100\%," Nat. Photonics 3, 297 (2009).

${ }^{4} \mathrm{~S}$. Gélinas et al., "Ultrafast long-range charge separation in organic semiconductor photovoltaic diodes," Science 343, 512 (2014).

${ }^{5}$ J.-L. Brédas, E. H. Sargent, and G. D. Scholes, "Photovoltaic concepts inspired by coherence effects in photosynthetic systems," Nat. Mater. 16, 35 (2016).

${ }^{6}$ N. Banerji, S. Cowan, M. Leclerc, E. Vauthey, and A. J. Heeger, "Exciton formation, relaxation, and decay in PCDTBT," J. Am. Chem. Soc. 132, 17459-17470 (2010).

${ }^{7}$ P. Prins et al., "High intrachain hole mobility on molecular wires of ladder-type poly(p-phenylenes)," Phys. Rev. Lett. 96, 146601 (2006).

${ }^{8}$ A. C. Jakowetz et al., "Visualizing excitations at buried heterojunctions in organic semiconductor blends," Nat. Mater. 16, 551 (2017).

${ }^{9}$ I. Hwang, S. Beaupré, M. Leclerc, and G. D. Scholes, "Ultrafast relaxation of charge-transfer excitons in low-bandgap conjugated copolymers," Chem. Sci. 3, 2270-2277 (2012)

${ }^{10}$ J. Guo, H. Ohkita, H. Benten, and S. Ito, "Near-IR femtosecond transient absorption spectroscopy of ultrafast polaron and triplet exciton formation in polythiophene films with different regioregularities," J. Am. Chem. Soc. 131, 16869-16880 (2009)

${ }^{11} \mathrm{R}$. Tautz et al., "Structural correlations in the generation of polaron pairs in low-bandgap polymers for photovoltaics," Nat. Commun. 3, 970 (2012).

${ }^{12}$ G. D. Scholes, "Long-range resonance energy transfer in molecular systems," Annu. Rev. Phys. Chem. 54, 57-87 (2003).
${ }^{13}$ V. Coropceanu et al., "Charge transport in organic semiconductors," Chem. Rev. 107, 926-952 (2007).

${ }^{14}$ P. B. Miranda, D. Moses, and A. J. Heeger, "Ultrafast photogeneration of charged polarons in conjugated polymers," Phys. Rev. B 64, 081201 (2001).

${ }^{15} \mathrm{P}$. Roy et al., "Ultrafast bridge planarization in donor- $\pi$-acceptor copolymers drives intramolecular charge transfer," Nat. Commun. 8, 1716 (2017).

${ }^{16} \mathrm{~F}$. Provencher et al., "Direct observation of ultrafast long-range charge separation at polymer-fullerene heterojunctions," Nat. Commun. 5, 4288 (2014).

${ }^{17}$ T. J. Magnanelli and A. E. Bragg, "Time-resolved Raman spectroscopy of polaron pair formation in poly(3-hexylthiophene) aggregates," J. Phys. Chem. Lett. 6, 438-445 (2015).

${ }^{18}$ A. De Sio et al., "Tracking the coherent generation of polaron pairs in conjugated polymers," Nat. Commun. 7, 13742 (2016).

${ }^{19} \mathrm{~S}$. M. Falke et al., "Coherent ultrafast charge transfer in an organic photovoltaic blend," Science 344, 1001 (2014).

${ }^{20}$ H. Tamura, I. Burghardt, and M. Tsukada, "Exciton dissociation at thiophene/fullerene interfaces: The electronic structures and quantum dynamics," J. Phys. Chem. C 115, 10205-10210 (2011).

${ }^{21}$ H. Tamura, R. Martinazzo, M. Ruckenbauer, and I. Burghardt, "Quantum dynamics of ultrafast charge transfer at an oligothiophene-fullerene heterojunction," J. Chem. Phys. 137, 22A540 (2012).

${ }^{22}$ G. D. Scholes, "Insights into excitons confined to nanoscale systems: Electronhole interaction, binding energy, and photodissociation," ACS Nano 2, 523-537 (2008).

${ }^{23}$ C. M. Pochas and F. C. Spano, "New insights on the nature of twodimensional polarons in semiconducting polymers: Infrared absorption in poly (3-hexylthiophene)," J. Chem. Phys. 140, 244902 (2014).

${ }^{24}$ N. E. Jackson, B. M. Savoie, T. J. Marks, L. X. Chen, and M. A. Ratner, “The next breakthrough for organic photovoltaics?," J. Phys. Chem. Lett. 6, 77-84 (2015).

${ }^{25}$ S.-H. Yeh, R. D. Hoehn, M. A. Allodi, G. S. Engel, and S. Kais, "Elucidation of near-resonance vibronic coherence lifetimes by nonadiabatic electronicvibrational state character mixing," Proc. Natl. Acad. Sci. U. S. A. 116, 18263 (2018).

${ }^{26}$ V. Tiwari, W. K. Peters, and D. M. Jonas, "Electronic resonance with anticorrelated pigment vibrations drives photosynthetic energy transfer outside the adiabatic framework," Proc. Natl. Acad. Sci. U. S. A. 110, 1203 (2013).

${ }^{27}$ S. Rafiq and G. D. Scholes, "Is back-electron transfer process in Betaine-30 coherent?," Chem. Phys. Lett. 683, 500-506 (2017).

${ }^{28}$ S. G. Boxer, "Stark realities," J. Phys. Chem. B 113, 2972-2983 (2009).

${ }^{29}$ S. D. Fried and S. G. Boxer, "Measuring electric fields and noncovalent interactions using the vibrational Stark effect," Acc. Chem. Res. 48, 998-1006 (2015).

${ }^{30}$ N. Blouin, A. Michaud, and M. Leclerc, "A low-bandgap poly(2,7-carbazole) derivative for use in high-performance solar cells," Adv. Mater. 19, 2295-2300 (2007).

${ }^{31}$ S. Beaupré and M. Leclerc, "PCDTBT: En route for low cost plastic solar cells," J. Mater. Chem. A 1, 11097-11105 (2013).

${ }^{32}$ C. H. Peters et al., "High efficiency polymer solar cells with long operating lifetimes," Adv. Energy Mater. 1, 491-494 (2011).

${ }^{33}$ N. Banerji et al., "Breaking down the problem: Optical transitions, electronic structure, and photoconductivity in conjugated polymer PCDTBT and in its separate building blocks," J. Phys. Chem. C 116, 11456-11469 (2012).

${ }^{34}$ L. G. Kaake et al., "Photoinduced charge generation in a molecular bulk heterojunction material,” J. Am. Chem. Soc. 134, 19828-19838 (2012).

${ }^{35} \mathrm{~J}$. Niklas et al., "Highly-efficient charge separation and polaron delocalization in polymer-fullerene bulk-heterojunctions: A comparative multi-frequency EPR and DFT study," Phys. Chem. Chem. Phys. 15, 9562-9574 (2013).

${ }^{36}$ S. Irgen-Gioro, A. P. Spencer, W. O. Hutson, and E. Harel, "Coherences of bacteriochlorophyll a uncovered using 3D-electronic spectroscopy," J. Phys. Chem. Lett. 9, 6077-6081 (2018).

${ }^{37}$ E. Harel, A. F. Fidler, and G. S. Engel, "Real-time mapping of electronic structure with single-shot two-dimensional electronic spectroscopy," Proc. Natl. Acad. Sci. U. S. A. 107, 16444 (2010). 
${ }^{38} \mathrm{E}$. Harel, "Zooming in on vibronic structure by lowest-value projection reconstructed 4D coherent spectroscopy,” J. Chem. Phys. 148, 194201 (2018).

${ }^{39} \mathrm{M}$. Tong et al., "Charge carrier photogeneration and decay dynamics in the poly(2,7-carbazole) copolymer PCDTBT and in bulk heterojunction composites with PC70BM," Phys. Rev. B 81, 125210 (2010).
${ }^{40}$ Y. Song, S. N. Clafton, R. D. Pensack, T. W. Kee, and G. D. Scholes, "Vibrational coherence probes the mechanism of ultrafast electron transfer in polymerfullerene blends," Nat. Commun. 5, 4933 (2014).

${ }^{41}$ S. Padgaonkar et al., "Molecular-orientation-dependent interfacial charge transfer in phthalocyanine/MoS2 mixed-dimensional heterojunctions," J. Phys. Chem. C 123, 13337-13343 (2019). 